\title{
Bifurcation analysis and dimension reduction of a predator-prey model for the L-H transition
}

Dam, Magnus; Brøns, Morten; Juul Rasmussen, Jens; Naulin, Volker; Xu, Guosheng

Published in:

Physics of Plasmas

Link to article, DOI:

$10.1063 / 1.4823719$

Publication date:

2013

Document Version

Publisher's PDF, also known as Version of record

Link back to DTU Orbit

Citation (APA):

Dam, M., Brøns, M., Juul Rasmussen, J., Naulin, V., \& Xu, G. (2013). Bifurcation analysis and dimension reduction of a predator-prey model for the L-H transition. Physics of Plasmas, 20(10), 102302. https://doi.org/10.1063/1.4823719

\section{General rights}

Copyright and moral rights for the publications made accessible in the public portal are retained by the authors and/or other copyright owners and it is a condition of accessing publications that users recognise and abide by the legal requirements associated with these rights.

- Users may download and print one copy of any publication from the public portal for the purpose of private study or research.

- You may not further distribute the material or use it for any profit-making activity or commercial gain

- You may freely distribute the URL identifying the publication in the public portal 


\section{AIP | Physics of Plasmas}

Bifurcation analysis and dimension reduction of a predator-prey model for the L-H transition

Magnus Dam, Morten Brøns, Jens Juul Rasmussen, Volker Naulin, and Guosheng Xu

Citation: Physics of Plasmas (1994-present) 20, 102302 (2013); doi: 10.1063/1.4823719

View online: http://dx.doi.org/10.1063/1.4823719

View Table of Contents: http://scitation.aip.org/content/aip/journal/pop/20/10?ver=pdfcov

Published by the AIP Publishing

AlP Re-register for Table of Content Alerts 


\title{
Bifurcation analysis and dimension reduction of a predator-prey model for the L-H transition
}

\author{
Magnus Dam, ${ }^{1}$ Morten Brøns, ${ }^{1}$ Jens Juul Rasmussen, ${ }^{2}$ Volker Naulin, ${ }^{2}$ and Guosheng $\mathrm{Xu}^{3}$ \\ ${ }^{1}$ Department of Applied Mathematics and Computer Science, Technical University of Denmark, \\ DK-2800 Kgs. Lyngby, Denmark \\ ${ }^{2}$ Association Euratom-DTU, Department of Physics, Technical University of Denmark, DTU Ris $\phi$ Campus, \\ DK-4000 Roskilde, Denmark \\ ${ }^{3}$ Institute of Plasma Physics, Chinese Academy of Sciences, Hefei 230031, China
}

(Received 28 June 2013; accepted 11 September 2013; published online 1 October 2013)

The L-H transition denotes a shift to an improved confinement state of a toroidal plasma in a fusion reactor. A model of the $\mathrm{L}-\mathrm{H}$ transition is required to simulate the time dependence of tokamak discharges that include the L-H transition. A 3-ODE predator-prey type model of the $\mathrm{L}-\mathrm{H}$ transition is investigated with bifurcation theory of dynamical systems. The analysis shows that the model contains three types of transitions: an oscillating transition, a sharp transition with hysteresis, and a smooth transition. The model is recognized as a slow-fast system. A reduced 2-ODE model consisting of the full model restricted to the flow on the critical manifold is found to contain all the same dynamics as the full model. This means that all the dynamics in the system is essentially 2-dimensional, and a minimal model of the L-H transition could be a 2-ODE model. C 2013 AIP Publishing LLC. [http://dx.doi.org/10.1063/1.4823719]

\section{INTRODUCTION}

The confinement of particles and energy and thereby the performance of a fusion reactor are strongly influenced by turbulent transport. ${ }^{1,2}$ The L- and H-modes are confinement states of a toroidal plasma, referring to states of low and high confinement, respectively. In the L-mode the transport is generally increasing when the input power is increased, until the edge heat flux exceeds a threshold value, where a transport barrier forms at the edge of the plasma and the plasma state enters the H-mode. ${ }^{3}$ The transition from the L- to the $\mathrm{H}$-mode is called the $\mathrm{L}-\mathrm{H}$ transition, and it is observed by spontaneously improved confinement properties of the plasma. The L-H transition was first observed experimentally in 1982 at the ASDEX tokamak ${ }^{4}$ and has since been routinely observed and controlled in fusion experiments.

Recent experiments, ${ }^{5,6}$ equipped with advanced diagnostics to provide spatial and temporal information, have revealed detailed information of the so-called L-I-H transition. The L-I-H transition takes place when the power input is slowly ramped up. The I-mode is an intermediate mode between the L- and H-mode, characterized by an oscillatory behavior. The I-mode is often referred to as the dithering phase, and it corresponds to the T-mode observed in predator-prey type models ${ }^{7-9}$ as considered in the present paper. The experiments revealed the causality in the interaction of turbulence, zonal flows, and mean flows. Zonal flows are generated by the turbulence through the Reynolds stress and form transport barriers. This in turn leads to steepening of the pressure gradient in the edge regime. The zonal flow is the predator and the turbulence the prey. A cyclic interaction appears with oscillating turbulent intensity and zonal flow strength. In each cycle the pressure gradient is stepwise increased. In turn this leads to the build up of a mean equilibrium flow induced by the pressure gradient through the radial force balance. Ultimately, the mean flow reaches a level sufficient for setting up the edge transport barrier sustaining the steep pressure gradient called the edge pedestal, and the plasma enters the H-mode.

Despite thorough investigations of the L-H transition it still lacks a first principle explanation. Models of the L-H transition might contribute to a better understanding of the underlying mechanisms. There exist several models ${ }^{6-10}$ consisting of systems of ordinary differential equations (ODEs), which try to describe the time evolution of key variables that characterize the L-H transition in different regimes. These models average over or ignore the spatial dependency attempting to achieve a minimal model that describes the most essential physics of the L-H transition. Most of these models are population models of the predator-prey type. Significant insight into the L-H transition dynamics has been gained from these simple population models. Other models ${ }^{11,12}$ include both a radial space coordinate and the time as independent variables, meaning they are systems of coupled partial differential equations.

In the present paper we analyze in detail the 3-ODE L-H transition model proposed in Ref. 7. The purpose of the paper is threefold. First we extend the bifurcation analysis in Ref. 8. We provide a systematic study of the structural changes of the bifurcation diagram as a function of the remaining five parameters in the system. Second, we discuss the statement in Ref. 13 that a proper model of the L-H transition should allow three types of transitions to occur: an oscillating transition, a sharp transition with hysteresis, and a smooth transition. It is claimed that a special three-parameter bifurcation is a necessary ingredient for all the transitions to occur. We use the findings from the bifurcation analysis to show that the model from Ref. 7 does allow the three types of transitions, even though it does not exhibit the special three-parameter bifurcation. Finally, we apply geometric singular perturbation theory to reduce the 3-ODE system to a 
2-ODE system that contains all the same dynamics. This shows that a 2-ODE system is sufficient for obtaining a minimal model of the L-H transition.

\section{THE 3-ODE L-H TRANSITION MODEL}

We consider the L-H transition model proposed in Ref. 7. The only independent variable in the model is the time $t$ making the model zero-dimensional in the physical space. The dependent variables in the model are the drift wave turbulence level $\mathcal{E}$, the shear of the zonal flow $V_{\mathrm{zf}}$, the gradient of the ion pressure $\mathcal{N}$, and the shear of the mean flow $V$. The model is a system of three coupled first order ODEs in the variables $\mathcal{E}, V_{\text {zf }}$, and $\mathcal{N}$ together with the algebraic equation $V=c_{3} \mathcal{N}^{2}$ relating the mean flow shear to the ion pressure gradient. The model can be formulated as the following 3-ODE system:

$$
\begin{gathered}
\frac{d}{d t} \mathcal{E}=\mathcal{E}\left(\mathcal{N}-a_{1} \mathcal{E}-a_{2} c_{3}^{2} \mathcal{N}^{4}-a_{3} V_{\mathrm{zf}}^{2}\right), \\
\frac{d}{d t} V_{\mathrm{zf}}=V_{\mathrm{zf}}\left(\frac{b_{1} \mathcal{E}}{1+b_{2} c_{3}^{2} \mathcal{N}^{4}}-b_{3}\right) \\
\frac{d}{d t} \mathcal{N}=Q(t)-\mathcal{N}\left(c_{1} \mathcal{E}+c_{2}\right)
\end{gathered}
$$

where $a_{i}, b_{i}, c_{i}, i=1,2,3$ are parameters. $Q$ represents the input power and may depend on time. All parameters and the function $Q$ are assumed to be positive. Equation (1a) describes the evolution of $\mathcal{E}$. The first term on the right-hand side represents that the pressure gradient $\mathcal{N}$ generates drift wave turbulence. The second term represents the nonlinear selfsaturation of drift waves. The third term represents the suppression of drift waves by the mean flow. The fourth term represents the suppression of drift waves by the zonal flow. Equation (1b) describes the evolution of $V_{\mathrm{zf}}$. The first term on the right-hand side represents the generation of zonal flow shear by Reynolds stress. The growth is inhibited by the mean flow shear, which is modeled by a factor $1 /\left(1+b_{2} V^{2}\right)$. The second term represents collisional damping of the zonal flow shear. Equation (1c) describes the evolution of $\mathcal{N}$. The first term on the right-hand side is the input power $Q$. The second term represents the turbulent diffusion of the profile by drift wave turbulence. The third term represents that the gradient decreases due to neoclassical transport.
The system (1) can be interpreted as a population model. In this interpretation $V_{\text {zf }}$ is a predator having $\mathcal{E}$ as prey and $\mathcal{E}$ is a predator having $\mathcal{N}$ as prey.

\section{A. Non-dimensionalizing the system}

The system (1) has a total of nine parameters in addition to the function $Q(t)$. To reduce the number of parameters, the system is non-dimensionalized by introducing new variables and time

$$
\begin{aligned}
& u=a_{1} a_{2}^{1 / 3} c_{3}^{2 / 3} \mathcal{E}, \quad v=a_{2}^{1 / 3} a_{3} c_{3}^{2 / 3} V_{\mathrm{zf}}^{2} \\
& w=a_{2}^{1 / 3} c_{3}^{2 / 3} \mathcal{N}, \quad s=a_{2}^{-1 / 3} c_{3}^{-2 / 3} t .
\end{aligned}
$$

This results in the following rescaled system:

$$
\left(\begin{array}{c}
\dot{u} \\
\dot{v} \\
\dot{w}
\end{array}\right)=F=\left(\begin{array}{l}
f \\
g \\
h
\end{array}\right)=\left(\begin{array}{c}
u\left(w-u-v-w^{4}\right) \\
\mu_{1} v\left(\frac{u}{1+\mu_{4} w^{4}}-\mu_{2}\right) \\
\mu_{5}\left(\sigma-w\left(1+\mu_{3} u\right)\right)
\end{array}\right),
$$

where the overdot denotes differentiation with respect to $s$. Five new dimensionless parameters and a new function $\sigma=\sigma(s)$ have been introduced. These are

$$
\begin{gathered}
\mu_{1}=\frac{2 b_{1}}{a_{1}}, \quad \mu_{2}=\frac{a_{1} a_{2}^{1 / 3} c_{3}^{2 / 3} b_{3}}{b_{1}}, \quad \mu_{3}=\frac{c_{1}}{a_{1} a_{2}^{1 / 3} c_{3}^{2 / 3} c_{2}}, \\
\mu_{4}=\frac{b_{2}}{a_{2}^{4 / 3} c_{3}^{2 / 3}}, \quad \mu_{5}=c_{2} a_{2}^{1 / 3} c_{3}^{2 / 3},
\end{gathered}
$$

and

$$
\sigma(s)=\frac{a_{2}^{1 / 3} c_{3}^{2 / 3}}{c_{2}} Q\left(a_{2}^{1 / 3} c_{3}^{2 / 3} s\right) .
$$

The phase space of the system (2) is $\mathcal{M}=\mathbb{R}_{+}^{3}$. We have chosen the same scaling as in Ref. 8, but we have defined the two new parameters $\mu_{3}, \mu_{5}$ and the scaled input power function $\sigma$ in a different way which will be more convenient for the dimension reduction in Sec. V.

\section{B. Sweeping rate influence}

We call the rate at which the heating power $\sigma$ changes in time, that is $d \sigma / d s$, the sweeping rate, and we consider how the sweeping rate can influence solutions of the nonautonomous system (2). Fig. 1 shows three examples of numerical (a)

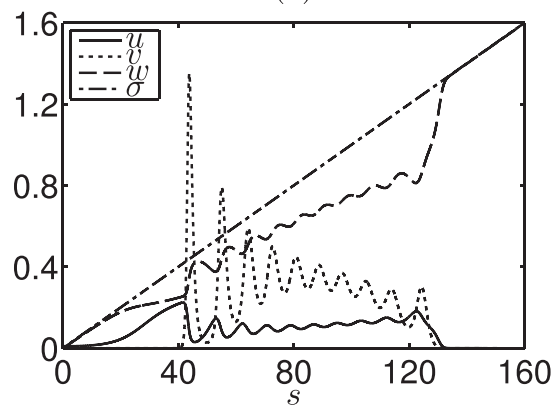

(b)

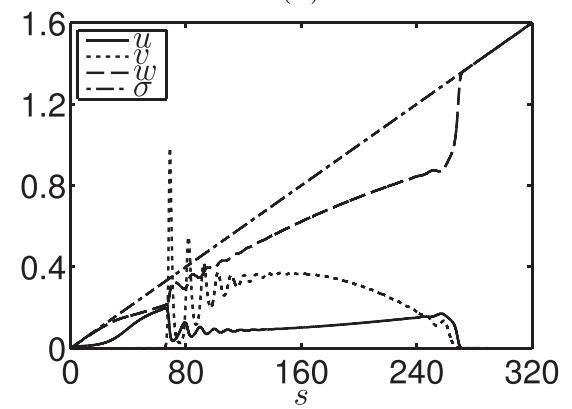

(c)

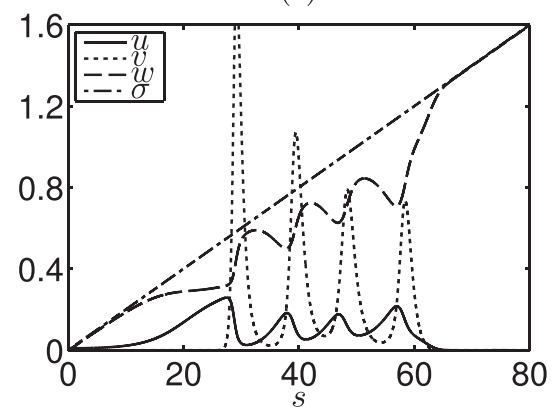

FIG. 1. Numerical solutions of the system (2) with the parameter values $\mu_{1}=18, \mu_{2}=0.08, \mu_{3}=2.8, \mu_{4}=1.8, \mu_{5}=8$, and the initial condition $u(0)=v(0)$ $=w(0)=0.01$ for three different sweeping rates: (a) $\sigma(s)=0.01 s$, (b) $\sigma(s)=0.005 s$, (c) $\sigma(s)=0.02 s$. The plots show the evolution of $u$, $v$, and $w$ as functions of time, $s$. The evolution of $\sigma$ has also been plotted for comparison. 
solutions to the system (2) with different sweeping rates. The parameter values are chosen to give a solution that passes through three different regimes: the L-mode, the T-mode, and the QH-mode. The L-mode is a state of low confinement, the T-mode a transient or intermediate state giving rise to dithering, and the QH-mode a state of high confinement.

By comparing the three plots in Fig. 1 we see that different sweeping rates result in significant differences in how the solution looks. In Fig. 1(a) a sweeping rate of $\sigma(s)=0.01 \mathrm{~s}$ was used, to obtain a solution with the desired oscillating behavior of the intermediate mode: The solution first spirals towards an equilibrium, but it then starts to spiral away from it again before an equilibrium state is obtained. In Fig. 1(b) the sweeping rate was lowered to $\sigma(s)=0.005 s$. The intermediate mode is no longer oscillating except at the transition to and from this mode: The solution spirals quickly into an equilibrium and stays in the equilibrium state until the transition to the QH-mode occurs. In Fig. 1(c) the sweeping rate was raised to $\sigma(s)=0.02 s$, and the intermediate mode now makes only a few oscillations with large amplitudes: The solution spirals slowly towards an equilibrium, but it never comes very near it before the transition to the QH-mode occurs. Even though the sweeping rate only was lowered and raised, respectively, by a factor of 2 , the behavior changed significantly. However, the qualitative behavior with the three phases, i.e., a low-confinement, an intermediate, and a high confinement phase, is unaffected by the sweeping rate.

\section{BIFURCATION ANALYSIS OF THE L-H TRANSITION MODEL}

We now assume that the heating power $\sigma$ develops sufficiently slowly in time such that we can ignore its timedependency and treat it as a constant parameter. Then the system (2) can be analyzed as an autonomous system. $\sigma$ will be treated as the main control parameter of the system.

A bifurcation analysis similar to the one given in the current section can be found in Ref. 8. We have here added information about how the intervals of existence and the stability of the equilibrium points depend on the parameters $\mu_{i}, i=1, \ldots, 5$. This will be necessary for the determination of the criteria for obtaining each of the three types of transitions in Sec. IV.

\section{A. Nullclines}

The nullclines of the system are the surfaces at which a single component of the velocity vanishes. Understanding the location of the nullclines is most useful to the analysis of the system. The $u$ - and the $v$-nullcline is each a union of two surfaces while the $w$-nullcline is a single surface

$$
\begin{aligned}
& \mathcal{N}_{u}=\{u=0\} \cup\left\{u=w\left(1-w^{3}\right)-v\right\}, \\
& \mathcal{N}_{v}=\{v=0\} \cup\left\{u=\mu_{2}\left(1+\mu_{4} w^{4}\right)\right\}, \\
& \mathcal{N}_{w}=\left\{w\left(1+\mu_{3} u\right)=\sigma\right\} .
\end{aligned}
$$

Since the plane $u=0$ belongs to the $u$-nullcline and the plane $v=0$ belongs to the $v$-nullcline, these two surfaces are invariant manifolds of the system, meaning that if the solution starts in one of these planes, it stays in that same plane for all time. This implies that solutions cannot cross these surfaces. In the plane $w=0$, we have $d w / d t \geq 0$, meaning that solutions starting in $\mathcal{M}$ cannot cross the plane $w=0$ in forward time. This makes the phase space $\mathcal{M}$ a forward invariant set.

All the nullcline surfaces are independent of $v$ except the surface $u=w\left(1-w^{3}\right)-v$ belonging to the $u$-nullcline. For each fixed $w$, curves on this surface decrease linearly in $v$. The nullclines are hard to visualize in the three-dimensional phase space, so instead the intersection curves of the nullclines with the plane $v=0$ (which is a part of $\mathcal{N}_{v}$ ) are shown in Fig. 2.

The intersection of all three nullclines, $\mathcal{S}=\mathcal{N}_{u} \cap \mathcal{N}_{v} \cap$ $\mathcal{N}_{w}$, consists of the equilibrium points. Since the plane $v=0$ belongs to the $v$-nullcline, any intersection between the $u$ nullcline and the $w$-nullcline in this plane is an equilibrium point. In Fig. 2 it can be seen that there are three equilibrium points in the plane $v=0$ which are labeled as $\mathrm{L}, \mathrm{H}$, and $\mathrm{QH}$. The projection of a fourth equilibrium, which is the only point where the three nullclines cross above the plane $v=0$, is marked with a T. This way of naming the equilibria is adopted from Ref. 8. The L-equilibrium represents a state of low confinement, the H-equilibrium a state of higher confinement, the QH-equilibrium represents a quiescent state of high confinement, while the T-equilibrium represents the transient or intermediate state giving rise to oscillations. We are interested in the transition from the $\mathrm{L}$ - to the $\mathrm{QH}$-equilibrium.

\section{B. Positions of equilibria}

For each of the four equilibrium points the position and the criteria for being inside $\mathcal{M}$ are determined. The equilibrium points of the system (2) are found by solving $F(u, v, w)=(0,0,0)^{\top}$. This equation cannot be fully solved analytically. Therefore we will for the L-, H-, and Tequilibrium just give the equations that determine the position of each equilibrium point.

\section{The position of the QH-equilibrium}

The QH-equilibrium is explicitly given by

$$
\left(u_{\mathrm{QH}}, v_{\mathrm{QH}}, w_{\mathrm{QH}}\right)=(0,0, \sigma) .
$$

The QH-equilibrium is inside $\mathcal{M}$ for all $\sigma \geq 0$.

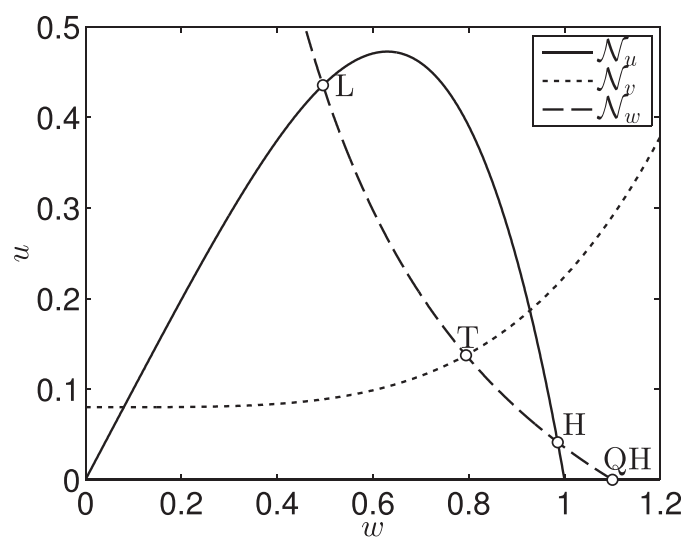

FIG. 2. Intersection curves of the nullclines in the plane $v=0$ for the parameter values $\mu_{1}=18, \mu_{2}=0.08, \mu_{3}=2.8, \mu_{4}=1.8, \mu_{5}=8$, and $\sigma=1.1$. The equilibrium points labeled $\mathrm{L}, \mathrm{H}$, and $\mathrm{QH}$ lies in the plane $v=0$, while the equilibrium point labeled $\mathrm{T}$ is projected onto the plane $v=0$. 


\section{The position of the L-equilibrium}

In Fig. 2 it is seen that the L-equilibrium is the first intersection point between the two curves $u=w\left(1-w^{3}\right)$ belonging to $\mathcal{N}_{u}$ and $w\left(1+\mu_{3} u\right)=\sigma$ belonging to $\mathcal{N}_{w}$ in the plane $v=0$. This means that the L-equilibrium is

$$
\left(u_{\mathrm{L}}, v_{\mathrm{L}}, w_{\mathrm{L}}\right)=\left(w_{\mathrm{L}}\left(1-w_{\mathrm{L}}^{3}\right), 0, w_{\mathrm{L}}\right),
$$

where $w_{\mathrm{L}}$ is the smallest of the two positive roots of the fifth order polynomial

$$
P(w)=\mu_{3} w^{2}\left(1-w^{3}\right)+w-\sigma .
$$

The polynomial $P(w)$ is plotted in Fig. 3 for four different values of $\sigma$. The smallest positive root of $P(w)$ is $w_{\mathrm{L}}$ while the greatest positive root of $P$ is $w_{\mathrm{H}}$. It is seen that the roots move closer to each other for $\sigma$ increasing from zero until the two equilibria coincide and disappear in a saddle-node bifurcation. The first derivative of the polynomial (5) is

$$
P^{\prime}(w)=\mu_{3} w\left(2-5 w^{3}\right)+1 .
$$

Since $P$ is concave downwards it always holds that

$$
P^{\prime}\left(w_{\mathrm{L}}\right)>0 \quad \text { and } \quad P^{\prime}\left(w_{\mathrm{H}}\right)<0 .
$$

The $w$-coordinate of the saddle-node bifurcation point is characterized by $P(w)=P^{\prime}(w)=0$, where the two positive roots of $P$ becomes a single-valued double root. For values of $\sigma$ higher than this there are no solutions to $P(w)=0$, meaning that neither the L-equilibrium or the H-equilibrium exists. For a fixed value of $\mu_{3}$ the $w$-coordinate of the saddlenode bifurcation point is characterized by $P^{\prime}\left(w_{\mathrm{sn}}\right)=0$. The value of $\sigma$ at the saddle-node bifurcation point is found by solving for $\sigma$ in the equation $P\left(w_{\mathrm{sn}}\right)=0$, which yields

$$
\sigma_{\mathrm{sn}}=\mu_{3} w_{\mathrm{sn}}^{2}\left(1-w_{\mathrm{sn}}^{3}\right)+w_{\mathrm{sn}}, \quad \mu_{3} \geq \frac{1}{3} .
$$

The condition $\mu_{3} \geq 1 / 3$ ensures that $w_{\text {sn }}$ lies in the interval $0 \leq w_{\mathrm{sn}} \leq 1$ such that $u_{\mathrm{sn}}=w_{\mathrm{sn}}\left(1-w_{\mathrm{sn}}^{3}\right) \geq 0$, which makes the saddle-node bifurcation occur inside $\mathcal{M}$. For $\mu_{3}<1 / 3$ the saddle-node bifurcation occurs outside of $\mathcal{M}$, but the

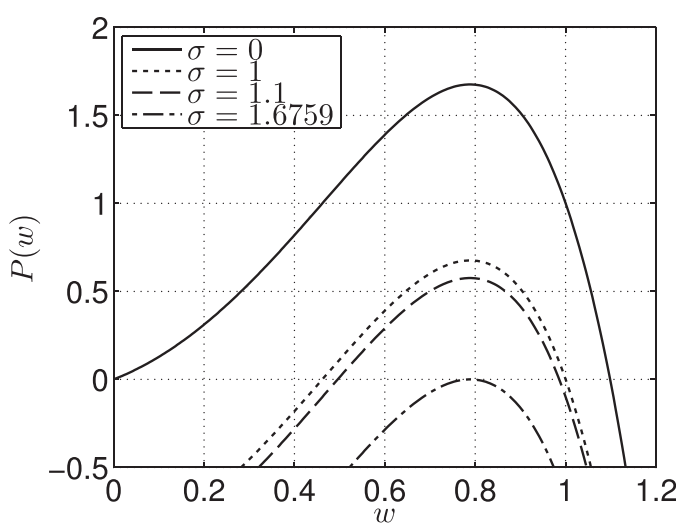

FIG. 3. Plot of the polynomial (5) with $\mu_{3}=2.8$ for three different values of $\sigma$. The first non-negative root of this polynomial is the value of $w_{\mathrm{L}}$, and the second positive root is the value of $w_{\mathrm{H}}$.
L-equilibrium is still inside $\mathcal{M}$ in the interval $0 \leq \sigma \leq 1$. So the L-equilibrium is inside $\mathcal{M}$ in the intervals

$$
\begin{cases}0 \leq \sigma \leq 1 & \text { for } \mu_{3}<\frac{1}{3} \\ 0 \leq \sigma \leq \sigma_{\mathrm{sn}} & \text { for } \mu_{3} \geq \frac{1}{3}\end{cases}
$$

\section{The position of the H-equilibrium}

The H-equilibrium is

$$
\left(u_{\mathrm{H}}, v_{\mathrm{H}}, w_{\mathrm{H}}\right)=\left(w_{\mathrm{H}}\left(1-w_{\mathrm{H}}^{3}\right), 0, w_{\mathrm{H}}\right),
$$

where $w_{\mathrm{H}}$ is the larger of the two positive roots of the polynomial (5). A necessary condition for the H-equilibrium to be inside $\mathcal{M}$ is that $0 \leq w_{\mathrm{H}} \leq 1$ to ensure that $u_{\mathrm{H}} \geq 0$. The H-equilibrium enters $\mathcal{M}$ at $\sigma_{\mathrm{tc} 2}=1$ and disappears in a saddle node bifurcation at $\sigma_{\mathrm{sn}}$ given by Eq. (8). So the $\mathrm{H}$-equilibrium is inside $\mathcal{M}$ in the interval

$$
1 \leq \sigma \leq \sigma_{\mathrm{sn}}, \quad \mu_{3} \geq \frac{1}{3} .
$$

The H-equilibrium is located outside of $\mathcal{M}$ for any value of $\sigma$ when $\mu_{3}<1 / 3$.

\section{The position of the T-equilibrium}

The T-equilibrium is the intersection point between the three surfaces $v=w\left(1-w^{3}\right)-u$ belonging to $\mathcal{N}_{u}, u=\mu_{2}$ $\left(1+\mu_{4} w^{4}\right)$ belonging to $\mathcal{N}_{v}$ and $w\left(1+\mu_{3} u\right)=\sigma$ belonging to $\mathcal{N}_{w}$. So the T-equilibrium is implicitly characterized by the point $\left(u_{\mathrm{T}}, v_{\mathrm{T}}, w_{\mathrm{T}}\right)$, where

$$
\begin{gathered}
u_{\mathrm{T}}=\mu_{2}\left(1+\mu_{4} w_{\mathrm{T}}^{4}\right), \\
v_{\mathrm{T}}=w_{\mathrm{T}}\left(1-w_{\mathrm{T}}^{3}\right)-u_{\mathrm{T}}, \\
w_{\mathrm{T}}=\frac{\sigma}{1+\mu_{3} u_{\mathrm{T}}} .
\end{gathered}
$$

By considering Fig. 2 we see that only the surface $u=\mu_{2}$ $\left(1+\mu_{4} w^{4}\right)$ belonging to $\mathcal{N}_{v}$ depends on $\mu_{2}$, and this surface moves upwards if $\mu_{2}$ is increased. If this surface is moved sufficiently upwards it will no longer intersect with the surface $u=w\left(1-w^{3}\right)-v$. So for the T-equilibrium to exist at all, $\mu_{2}$ must be sufficiently small. To determine exactly how small $\mu_{2}$ must be we consider the $v=0$ plane where we first determine the $w$-coordinate at which the two curves $u=w\left(1-w^{3}\right)$ belonging to $\mathcal{N}_{u}$ and $u=\mu_{2}\left(1+\mu_{4} w^{4}\right)$ belonging to $\mathcal{N}_{v}$ have the same slope. By differentiating these functions with respect to $w$ we see that the slope of the first curve is $d u / d w=1-4 w^{3}$, while the slope of the second curve is $d u / d w=4 \mu_{2} \mu_{4} w^{3}$. So these curves have the same slope at

$$
w_{0}=\frac{1}{\left(4\left(1+\mu_{2} \mu_{4}\right)\right)^{1 / 3}} .
$$

For the T-equilibrium to exist the $u$-coordinate of the T-equilibrium must at $w=w_{0}$ be smaller than the $u$-coordinate 
of the L-equilibrium at $w=w_{0}$, that is, $\left.u_{\mathrm{T}}\right|_{w_{\mathrm{T}}=w_{0}} \leq\left. u_{\mathrm{L}}\right|_{w_{\mathrm{L}}=w_{0}}$. By inserting the expressions for $u_{\mathrm{L}}$ from Eq. (4) and $u_{\mathrm{T}}$ from Eq. (10a) this condition becomes $\mu_{2}\left(1+\mu_{4} w_{0}^{4}\right) \leq w_{0}\left(1-w_{0}^{3}\right)$. By inserting the expression (11) for $w_{0}$ into this inequality and rearranging, the condition for the $\mathrm{T}$-equilibrium to exist for some value of $\sigma$ becomes $\mu_{2} \leq \mu_{2,0}$, where $\mu_{2,0}$ is the only positive solution to the equation

$$
256\left(1+\mu_{2} \mu_{4}\right) \mu_{2}^{3}-27=0 .
$$

Now assume that $\mu_{2} \leq \mu_{2,0}$. Then there exists an interval of $\sigma$ for which the T-equilibrium is inside $\mathcal{M}$. A necessary condition for the T-equilibrium to be inside $\mathcal{M}$ is that $v_{\mathrm{T}} \geq 0$. In each of the endpoints of the interval of $\sigma$ for which the T-equilibrium is inside $\mathcal{M}$ we have $v_{\mathrm{T}}=0$, and Eq. (10) that characterizes the T-equilibrium becomes identical to the one that characterizes the L-equilibrium and the H-equilibrium, respectively. For the lowest value of $\sigma$ for which the T-equilibrium is in $\mathcal{M}$, it coincides with the L-equilibrium. This occurs at $\sigma_{\mathrm{tc} 1}=w_{\mathrm{tc} 1}\left(1+\mu_{3} u_{\mathrm{tc} 1}\right)$ in the point

$$
\left(u_{\mathrm{tc} 1}, v_{\mathrm{tc} 1}, w_{\mathrm{tc} 1}\right)=\left(w_{\mathrm{tc} 1}\left(1-w_{\mathrm{tc} 1}^{3}\right), 0, w_{\mathrm{tc} 1}\right),
$$

where $w_{\mathrm{tc} 1}$ is the smallest positive solution to the equation

$$
\left(1+\mu_{2} \mu_{4}\right) w^{4}-w+\mu_{2}=0 .
$$

For the greatest value of $\sigma$ for which the T-equilibrium is in $\mathcal{M}$ it coincides with either the L-equilibrium once again or with the H-equilibrium. In either case it occurs at $\sigma_{\mathrm{tc} 3}=w_{\mathrm{tc} 3}$ $\left(1+\mu_{3} u_{\mathrm{tc} 3}\right)$ in the point

$$
\left(u_{\mathrm{tc} 3}, v_{\mathrm{tc} 3}, w_{\mathrm{tc} 3}\right)=\left(w_{\mathrm{tc} 3}\left(1-w_{\mathrm{tc} 3}^{3}\right), 0, w_{\mathrm{tc} 3}\right),
$$

where $w_{\text {tc3 }}$ is the greatest solution to Eq. (12). So the T-equilibrium is inside $\mathcal{M}$ for

$$
\sigma_{\mathrm{tc} 1} \leq \sigma \leq \sigma_{\mathrm{tc} 3}, \quad \mu_{2} \leq \mu_{2,0}
$$

We will now determine a condition for whether the T-equilibrium coincides with the L-equilibrium or the $\mathrm{H}$-equilibrium as it leaves $\mathcal{M}$ at $\sigma=\sigma_{\text {tc } 3}$. As we will see, the L-equilibrium changes stability each time it coincides with the T-equilibrium. If the T-equilibrium coincides with the L-equilibrium as it leaves $\mathcal{M}$ the L-equilibrium becomes stable again, and we can have a back-transition to the L-mode after the dithering phase. If $\mu_{3}<1 / 3$ the $\mathrm{H}$-equilibrium is outside of $\mathcal{M}$ for all values of $\sigma$, and the T-equilibrium will coincide twice with the L-equilibrium. Therefore, assume $\mu_{3} \geq 1 / 3$ such that $w_{\text {sn }} \leq 1$. Then the T-equilibrium can be made to coincide with the saddle-node bifurcation by requiring that $u_{\mathrm{T}}$ is equal to $u_{\mathrm{sn}}=w_{\mathrm{sn}}\left(1-w_{\mathrm{sn}}^{3}\right)$ when $w_{\mathrm{T}}=w_{\mathrm{sn}}$. This implies that $\mu_{2}$ and $\mu_{4}$ must satisfy the relation

$$
\mu_{2}=\frac{w_{\mathrm{sn}}\left(1-w_{\mathrm{sn}}^{3}\right)}{1+\mu_{4} w_{\mathrm{sn}}^{4}} \equiv \mu_{2, \mathrm{c}} .
$$

Since the solution to $P\left(w_{\mathrm{sn}}\right)=0$ does not depend on the value of $\mu_{2}$ the three equilibrium points $\mathrm{L}, \mathrm{H}$, and $\mathrm{T}$ will for $\mu_{2}=\mu_{2, \mathrm{c}}$ all coincide at the same value of $\sigma$ for which the saddle-node bifurcation was found to occur. For $\mu_{2}>\mu_{2, c}$ the T-equilibrium coincides with the L-equilibrium in a transcritical bifurcation as the T-equilibrium leaves $\mathcal{M}$, and if $\mu_{2}<\mu_{2, \mathrm{c}}$ the T-equilibrium instead coincides with the $\mathrm{H}$ equilibrium as it leaves $\mathcal{M}$.

\section{Stability of equilibria}

In order to determine the stability of the four equilibrium points we linearize the system (2) around each equilibrium point. It will be used that the Jacobian matrix of the system (2) in a general point $(u, v, w)$ is

$$
\mathcal{D} F(u, v, w)=\left(\begin{array}{lll}
f_{u} & f_{v} & f_{w} \\
g_{u} & g_{v} & g_{w} \\
h_{u} & h_{v} & h_{w}
\end{array}\right)=\left(\begin{array}{ccc}
w-2 u-w^{4}-v & -u & u\left(1-4 w^{3}\right) \\
\frac{\mu_{1} v}{1+\mu_{4} w^{4}} & \mu_{1}\left(\frac{u}{1+\mu_{4} w^{4}}-\mu_{2}\right) & -\frac{4 \mu_{1} \mu_{4} u v w^{3}}{\left(1+\mu_{4} w^{4}\right)^{2}} \\
-\mu_{3} \mu_{5} w & 0 & -\mu_{5}\left(1+\mu_{3} u\right)
\end{array}\right) .
$$

\section{Stability of the $Q H-$ equilibrium}

The Jacobian matrix (14) evaluated in the QHequilibrium (3) is given by

$$
\mathcal{D} F(0,0, \sigma)=\left(\begin{array}{ccc}
\sigma\left(1-\sigma^{3}\right) & 0 & 0 \\
0 & -\mu_{1} \mu_{2} & 0 \\
-\mu_{3} \mu_{5} \sigma & 0 & -\mu_{5}
\end{array}\right) .
$$

The eigenvalues are

$$
\lambda_{1}=\sigma\left(1-\sigma^{3}\right), \quad \lambda_{2}=-\mu_{1} \mu_{2}, \quad \lambda_{3}=-\mu_{5} .
$$

The eigenvalues $\lambda_{2}$ and $\lambda_{3}$ are always negative while the sign of $\lambda_{1}$ depends on the value of $\sigma$. We have

$$
\left\{\begin{array}{l}
\lambda_{1}>0 \text { for } \quad 0<\sigma<1 \\
\lambda_{1}<0 \text { for } \quad \sigma>1
\end{array}\right.
$$

So the QH-equilibrium is a saddle and thus unstable for $\sigma<1$, and it is a stable node for $\sigma>1$. The $\mathrm{QH}$-equilibrium coincides with the $\mathrm{H}$-equilibrium in a transcritical bifurcation at $\sigma_{\mathrm{tc} 2}=1$.

\section{Stability of the L-equilibrium}

Now consider the L-equilibrium. Define new coordinates $(\delta u, \delta v, \delta w)$ centered at the L-equilibrium

$$
u=u_{\mathrm{L}}+\delta u, \quad v=0+\delta v, \quad w=w_{\mathrm{L}}+\delta w .
$$


The system (2) linearized around the L-equilibrium then becomes

$$
\begin{gathered}
\dot{\delta} u=-u_{\mathrm{L}} \delta u-u_{\mathrm{L}} \delta v+u_{\mathrm{L}}\left(1-4 w_{\mathrm{L}}^{3}\right) \delta w \\
\dot{\delta} v=\mu_{1}\left(\frac{u_{\mathrm{L}}}{1+\mu_{4} w_{\mathrm{L}}^{4}}-\mu_{2}\right) \delta v, \\
\dot{\delta} w=-\mu_{3} \mu_{5} w_{\mathrm{L}} \delta u-\mu_{5}\left(1+\mu_{3} u_{\mathrm{L}}\right) \delta w .
\end{gathered}
$$

The eigenvalues of the Jacobian matrix of the system (15) must be determined numerically. However, the stability of the L-equilibrium can still be determined analytically. The evolution of $\delta v$ depends only on $\delta v$ itself, so we can treat this direction independently of the other two directions. We see that

$$
\begin{cases}\dot{\delta} v<0 & \text { for } \quad u_{\mathrm{L}}<\mu_{2}\left(1+\mu_{4} w_{\mathrm{L}}^{4}\right) \\ \dot{\delta} v>0 & \text { for } \quad u_{\mathrm{L}}>\mu_{2}\left(1+\mu_{4} w_{\mathrm{L}}^{4}\right)\end{cases}
$$

So $\dot{\delta} v<0$ when the equilibrium point is located below the curve $u=\mu_{2}\left(1+\mu_{4} w^{4}\right)$ belonging to $\mathcal{N}_{v}$ and $\dot{\delta} v>0$ when the equilibrium point is located above this curve (see Fig. 2). So the L-equilibrium changes stability in the direction transverse to the plane $v=0$ each time it coincides with the T-equilibrium.

If $\mu_{2}>\mu_{2,0}$ the T-equilibrium does not exist, and the L-equilibrium is always located below the curve $u=\mu_{2}$ $\left(1+\mu_{4} w^{4}\right)$, making it attracting in the direction transverse to the plane $v=0$. If $\mu_{2}$ lies in the interval $\mu_{2, \mathrm{c}}<\mu_{2}<\mu_{2,0}$ the L-equilibrium coincides with the T-equilibrium twice with the L-equilibrium changing its transverse stability each time. If $\mu_{2}<\mu_{2, c}$ the T-equilibrium coincides with the L-equilibrium only once, by which the L-equilibrium becomes repelling in the direction transverse to the plane $v=0$.

We now assume $\delta v=0$ in Eq. (15) and consider the remaining two-dimensional system

$$
\left(\begin{array}{c}
\dot{\delta} u \\
\dot{\delta} w
\end{array}\right)=\left(\begin{array}{cc}
-u_{\mathrm{L}} & u_{\mathrm{L}}\left(1-4 w_{\mathrm{L}}^{3}\right) \\
-\mu_{3} \mu_{5} w_{\mathrm{L}} & -\mu_{5}\left(1+\mu_{3} u_{\mathrm{L}}\right)
\end{array}\right)\left(\begin{array}{c}
\delta u \\
\delta w
\end{array}\right) .
$$

Define $\tau$ to be the trace and $\delta$ to be the determinant of the system matrix of the reduced linearized system (16)

$$
\begin{gathered}
\tau=-\left(\left(1+\mu_{3} \mu_{5}\right) u_{\mathrm{L}}+\mu_{5}\right), \\
\delta=\mu_{5} u_{\mathrm{L}}\left(\mu_{3} w_{\mathrm{L}}\left(2-5 w_{\mathrm{L}}^{3}\right)+1\right) .
\end{gathered}
$$

Since $\tau<0$ one of the eigenvalues is always negative. The other eigenvalue is negative if and only if $\delta>0$, that is $\mu_{3} w_{\mathrm{L}}\left(2-5 w_{\mathrm{L}}^{3}\right)+1>0$, which becomes the condition for the L-equilibrium being stable in the plane $v=0$. By comparing with Eq. (6) this condition can be written as $P^{\prime}\left(w_{\mathrm{L}}\right)>0$. Then from Eq. (7) we conclude that inside $\mathcal{M}$ the L-equilibrium is always stable in the plane $v=0$. So the L-equilibrium is a stable node when it is attracting in the direction transverse to the plane $v=0$, and it is a saddle and thus unstable when it is repelling in the transverse direction.

\section{Stability of the H-equilibrium}

We now consider the H-equilibrium. Define new coordinates $(\delta u, \delta v, \delta w)$ centered at the H-equilibrium

$$
u=u_{\mathrm{H}}+\delta u, \quad v=0+\delta v, \quad w=w_{\mathrm{H}}+\delta w .
$$

The system (2) linearized around the H-equilibrium then becomes

$$
\begin{gathered}
\dot{\delta} u=-u_{\mathrm{H}} \delta u-u_{\mathrm{H}} \delta v+u_{\mathrm{H}}\left(1-4 w_{\mathrm{H}}^{3}\right) \delta w \\
\dot{\delta} v=\mu_{1}\left(\frac{u_{\mathrm{H}}}{1+\mu_{4} w_{\mathrm{H}}^{4}}-\mu_{2}\right) \delta v \\
\dot{\delta} w=-\mu_{3} \mu_{5} w_{\mathrm{H}} \delta u-\mu_{5}\left(1+\mu_{3} u_{\mathrm{H}}\right) \delta w .
\end{gathered}
$$

As for the L-equilibrium, the evolution of $\delta v$ only depends on $\delta v$ itself, so we can treat this direction independently of the other two directions. We see that

$$
\left\{\begin{array}{lll}
\dot{\delta} v<0 & \text { for } & u_{\mathrm{H}}<\mu_{2}\left(1+\mu_{4} w_{\mathrm{H}}^{4}\right) \\
\dot{\delta} v>0 & \text { for } & u_{\mathrm{H}}>\mu_{2}\left(1+\mu_{4} w_{\mathrm{H}}^{4}\right)
\end{array}\right.
$$

So $\dot{\delta} v<0$ when the H-equilibrium is located below the curve $u=\mu_{2}\left(1+\mu_{4} w^{4}\right)$ belonging to $\mathcal{N}_{v}$ and $\dot{\delta} v>0$ when the H-equilibrium is located above this $v$-nullcline (see Fig. 2). This means the H-equilibrium changes stability in the direction transverse to the plane $v=0$ when it coincides with the T-equilibrium. If $\mu_{2} \geq \mu_{2, c}$ the $\mathrm{H}$-equilibrium never coincides with the T-equilibrium, and it is therefore repelling in the direction transverse to the plane $v=0$ for all values of $\sigma$. When $\mu_{2}<\mu_{2, \mathrm{c}}$ the H-equilibrium coincides with the T-equilibrium once, by which it becomes attracting in the direction transverse to the plane $v=0$.

We now assume $\delta v=0$ in Eq. (17) and consider the remaining two-dimensional system

$$
\left(\begin{array}{c}
\dot{\delta} u \\
\dot{\delta} w
\end{array}\right)=\left(\begin{array}{cc}
-u_{\mathrm{H}} & u_{\mathrm{H}}\left(1-4 w_{\mathrm{H}}^{3}\right) \\
-\mu_{3} \mu_{5} w_{\mathrm{H}} & -\mu_{5}\left(1+\mu_{3} u_{\mathrm{H}}\right)
\end{array}\right)\left(\begin{array}{c}
\delta u \\
\delta w
\end{array}\right) .
$$

Let $\tau$ be the trace and $\delta$ the determinant of the system matrix of the reduced linearized system (18)

$$
\begin{aligned}
& \tau=-\left(\left(1+\mu_{3} \mu_{5}\right) u_{\mathrm{H}}+\mu_{5}\right), \\
& \delta=\mu_{5} u_{\mathrm{H}}\left(\mu_{3} w_{\mathrm{H}}\left(2-5 w_{\mathrm{H}}^{3}\right)+1\right) .
\end{aligned}
$$

Since $\tau<0$ one of the eigenvalues is always negative. The other eigenvalue is negative if and only if $\delta>0$, that is, $\mu_{3} w_{\mathrm{H}}\left(2-5 w_{\mathrm{H}}^{3}\right)+1>0$. This is the condition for the H-equilibrium being stable in the plane $v=0$. By comparing with Eq. (6) we see this condition can be written as $P^{\prime}\left(w_{\mathrm{H}}\right)>0$. From Eq. (7) we see that inside $\mathcal{M}$ the $\mathrm{H}$-equilibrium always has one positive eigenvalue in the plane $v=0$, making it a saddle point. This means the H-equilibrium is always unstable.

\section{Stability of the T-equilibrium}

The Jacobian matrix (14) evaluated in the T-equilibrium (10) is given by 


$$
\mathcal{D} F\left(u_{\mathrm{T}}, v_{\mathrm{T}}, w_{\mathrm{T}}\right)=\left(\begin{array}{ccc}
-u_{\mathrm{T}} & -u_{\mathrm{T}} & -u_{\mathrm{T}}\left(4 w_{\mathrm{T}}^{3}-1\right) \\
\frac{\mu_{1} \mu_{2} v_{\mathrm{T}}}{u_{\mathrm{T}}} & 0 & -\frac{4 \mu_{1} \mu_{2}^{2} \mu_{4} v_{\mathrm{T}} w_{\mathrm{T}}^{3}}{u_{\mathrm{T}}} \\
-\mu_{3} \mu_{5} w_{\mathrm{T}} & 0 & -\frac{\mu_{5} \sigma}{w_{\mathrm{T}}}
\end{array}\right) .
$$

Define $\tau$ to be the trace, $\delta$ to be the determinant and $\zeta$ to be the sum of the principal minors of the Jacobian matrix (19)

$$
\begin{aligned}
& \tau=-\left(u_{\mathrm{T}}+\frac{\mu_{5} \sigma}{w_{\mathrm{T}}}\right) \\
& \zeta=\frac{\mu_{5} \sigma u_{\mathrm{T}}}{w_{\mathrm{T}}}-\mu_{3} \mu_{5} u_{\mathrm{T}} w_{\mathrm{T}}\left(4 w_{\mathrm{T}}^{3}-1\right)+\mu_{1} \mu_{2} v_{\mathrm{T}}, \\
& \delta=-\mu_{1} \mu_{2} \mu_{5} v_{\mathrm{T}}\left(\frac{\sigma}{w_{\mathrm{T}}}+4 \mu_{2} \mu_{3} \mu_{4} w_{\mathrm{T}}^{4}\right) .
\end{aligned}
$$

We notice that $\tau<0$ and $\delta \leq 0$ with $\delta=0 \Longleftrightarrow v_{\mathrm{T}}=0$, while $\zeta$ can be both negative, zero, or positive. The characteristic polynomial written in terms of $\tau, \delta$, and $\zeta$ is given by

$$
p(\lambda)=\lambda^{3}-\tau \lambda^{2}+\zeta \lambda-\delta .
$$

A third-order polynomial with real coefficients always has at least one real root while the other two can either both be real or they can be complex conjugates. The roots can be found by numerically solving $p(\lambda)=0$ for $\lambda \cdot p(0)=-\delta$, so $\lambda=0$ is an eigenvalue for the T-equilibrium if and only if $v_{\mathrm{T}}=0$. For this single vanishing eigenvalue a transcritical bifurcation occurs. Now assume that $\lambda_{1}=0$ such that $v_{\mathrm{T}}=0$. It can be shown ${ }^{14}$ the condition for having a second vanishing eigenvalue is $P^{\prime}\left(w_{\mathrm{T}}\right)=0$. Since $P^{\prime}(w)=0$ also is the condition for the saddle-node bifurcation of the $\mathrm{L}$ and $\mathrm{H}$ equilibrium, there can only be two vanishing eigenvalues when these three equilibria coincide. This occurs when $\mu_{2}$ and $\mu_{4}$ satisfy Eq. (13) and $\sigma=\sigma_{\mathrm{sn}}$.

A pure imaginary eigenvalue is also possible. For a real number $\omega$ we find that $p(i \omega)=0$ if and only if $\omega^{2}=\delta / \tau=\zeta$. This can be fulfilled, so an Andronov-Hopf bifurcation occurs when

$$
\tau \zeta-\delta=0 .
$$

To check when the T-equilibrium is stable, we use the Routh-Hurwitz criterion. Assume $v_{\mathrm{T}}>0$. Then $\tau<0$ and $\delta<0$ is fulfilled for all parameter values while $\zeta$ can be both positive, zero, or negative. Since $\tau \zeta-\delta<0 \Rightarrow \zeta>0$ and $\zeta<0 \Rightarrow \tau \zeta-\delta>0$ the T-equilibrium is always stable for $\tau \zeta-\delta<0$ and unstable for $\tau \zeta-\delta>0$. So the T-equilibrium only changes stability as the Andronov-Hopf bifurcation occurs

\section{THREE TYPES OF TRANSITIONS}

After analyzing the system (2) we are now able to show that each of the three types of transitions, ${ }^{13}$ i.e., an oscillating transition, a sharp transition with hysteresis, and a smooth transition without hysteresis, can be achieved by an appropriate choice of parameter values.

In Fig. 4 we consider a bifurcation diagram for each of these three types of transitions. In the bifurcation diagrams all three coordinates $(u, v, w)$ of all equilibrium points are shown as functions of $\sigma$. Coordinates of stable equilibria are shown as solid curves, and unstable equilibria are shown as dashed curves. Each of the three coordinates has its own color, but there are no indicated distinctions between the L-, $\mathrm{H}-, \mathrm{T}-$, or $\mathrm{QH}-$ equilibrium. The expressions for the positions of the QH-equilibrium (3), L-equilibrium (4), H-equilibrium (9), and T-equilibrium (10) can be compared with the bifurcation diagram to identify which set of curves that correspond to each equilibrium point. The $\mathrm{QH}$-equilibrium has its $u$ - and $v$-coordinates equal to zero for all values of $\sigma$. This means there is neither zonal flow or turbulence in this mode. The L-equilibrium and the $\mathrm{H}$-equilibrium has their $v$-coordinate equal to zero for all values of $\sigma$, meaning there is no zonal flow in these modes. Because there are so many equilibrium point coordinates equal to zero, it is not possible to see these or their stability in the bifurcation diagrams, because the curves are plotted on top of each other.

According to Ref. 13 any candidate for an L-H transition should contain a special codimension-three bifurcation, which is the collision of a cusp bifurcation and a TakensBogdanov bifurcation, to ensure that it can reproduce all the three types of transitions. We did not find such a codimension-three bifurcation. Instead the three types of (a)

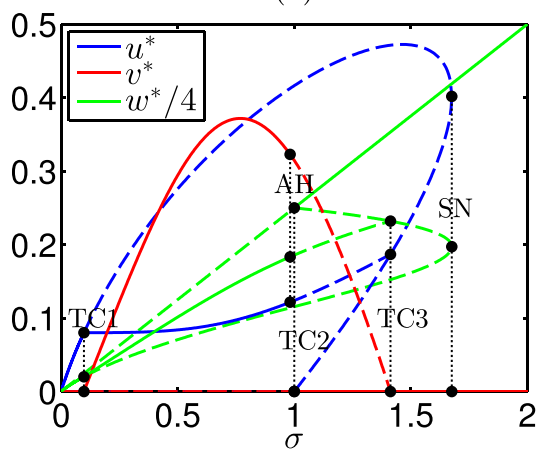

(b)

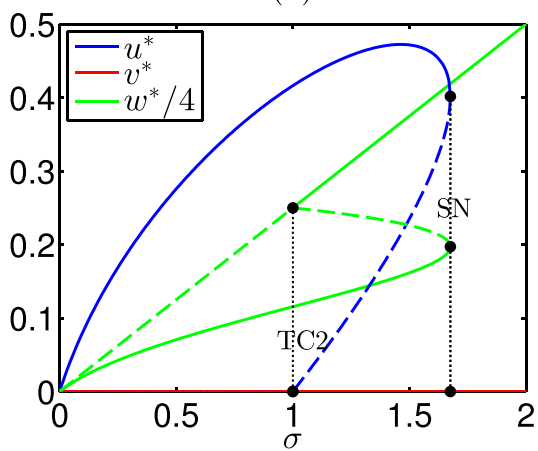

(c)

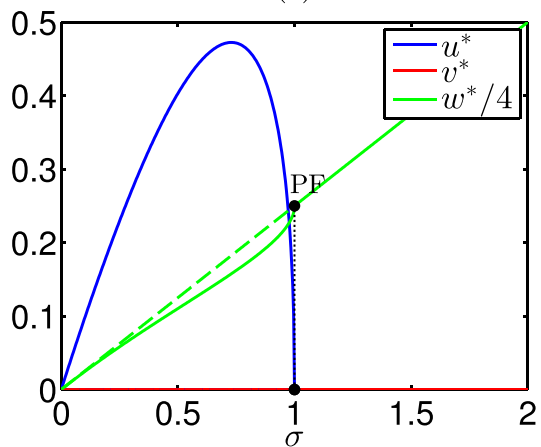

FIG. 4. Bifurcation diagrams showing the three distinct types of transitions: (a) an oscillating transition where $\mu_{2}=0.08, \mu_{3}=2.8$; (b) a sharp transition allowing hysteresis where $\mu_{2}=0.4, \mu_{3}=2.8$; (c) a smooth transition where $\mu_{2}=0.4, \mu_{3}=1 / 3$. Stable equilibria are marked with solid lines and unstable equilibria with dashed lines. For all three diagrams $\mu_{1}=18, \mu_{4}=1.8$, and $\mu_{5}=8$. 
transitions originate from an Andronov-Hopf bifurcation and the unfolding of a pitchfork bifurcation.

\section{A. The oscillating transition}

There are two conditions on the parameters for obtaining an oscillating transition:

(a) Ensuring that the T-equilibrium enters and leaves $\mathcal{M}$ at some point requires $\mu_{2} \leq \mu_{2,0}$.

(b) Ensuring a $\mathrm{L} \rightarrow \mathrm{T} \rightarrow \mathrm{QH}$ transition instead of a $\mathrm{L} \rightarrow$ $\mathrm{T} \rightarrow \mathrm{L} \rightarrow \mathrm{QH}$ transition requires $\mu_{2} \leq \mu_{2, \mathrm{c}}$ and $\mu_{3} \geq 1 / 3$.

The second condition may not be very important since the back-transition to the L-mode in simulations only lasts for a very short period of time. Fig. 4(a) shows a bifurcation diagram where these two conditions are fulfilled. There are a total of five different bifurcations that occur when $\sigma$ is varied in the interval $[0,2]$. The T-equilibrium coincides with the L-equilibrium in a transcritical bifurcation at $\sigma_{\mathrm{tc} 1}$ marked with TC1 on the diagram, the Andronov-Hopf bifurcation of the T-equilibrium occurs at $\sigma_{\mathrm{AH}}$ marked with $\mathrm{AH}$, the $\mathrm{H}$ equilibrium coincides with the $\mathrm{QH}$-equilibrium in a transcritical bifurcation at $\sigma_{\mathrm{tc} 2}$ marked with TC2, the T-equilibrium coincides with the H-equilibrium in a transcritical bifurcation at $\sigma_{\mathrm{tc} 3}$ marked with $\mathrm{TC} 3$, and the L- and H-equilibrium merge and disappear in a saddle-node bifurcation at $\sigma_{\mathrm{sn}}$ marked with SN on the diagram.

Depending on the parameters $\mu_{i}, i=1, \ldots, 5$ we can either have $\sigma_{\mathrm{AH}} \leq 1$, which is the situation in Fig. 4(a), or we can have $\sigma_{\mathrm{AH}}>1$. If $\sigma_{\mathrm{AH}}>1$ there exists an interval of bistability of the T- and the QH-equilibrium. This allows hysteresis for the T-QH transition, as discussed in Ref. 8. The interval of bistability is relatively small, so it exists only in a short time interval when $\sigma$ is swept. This means the T-QH hysteresis is unimportant. When the T-QH bistability is present the basins of attraction of the T- and the QH-equilibrium is separated by the 2-dimensional stable manifold of the H-equilibrium. Based on numerical investigations Ref. 8 suggests that the hysteresis is sensitive to initial conditions. To clarify, only solutions with initial conditions on the stable manifold of the H-equilibrium exhibit sensitive dependence on initial conditions in the sense that an arbitrarily small perturbation may move the state to any side of the separating manifold. Initial conditions away from this manifold do not have this property.

Fig. 5(a) shows the corresponding numerical solution of the system (2). By comparing the solution in Fig. 5(a) with the corresponding bifurcation diagram in Fig. 4(a) it can be seen that the solution first follows the L-equilibrium, and then it spirals towards the stable T-equilibrium and away from it again some time after it has become unstable at the Andronov-Hopf bifurcation. Then it moves to the $\mathrm{QH}-$ equilibrium.

\section{B. The sharp transition}

There are two conditions on the parameters for obtaining a sharp transition with hysteresis:

(a) Ensuring that the T-equilibrium never enters the phase space $\mathcal{M}$ requires $\mu_{2}>\mu_{2,0}$.

(b) Ensuring that the saddle-node bifurcation occurs inside the phase space $\mathcal{M}$ requires $\mu_{3}>1 / 3$.

Fig. 4(b) shows a bifurcation diagram with these conditions fulfilled. The number of bifurcations are now limited to two; the transcritical bifurcation that occurs as the H-equilibrium coincides with the QH-equilibrium marked with $\mathrm{TC} 2$, and the saddle-node bifurcation of the L- and H-equilibrium marked with SN on the diagram. Fig. 5(b) shows a corresponding numerical solution to the system (2). Since the T-equilibrium was the only equilibrium not located in the plane $v=0$ all dynamics now occurs in the plane $v=0$. So the transition occurs without generation of zonal flow. The transition from the L-mode to the QH-mode is sharp and occurs just after the saddle-node bifurcation. In the bifurcation diagram it can be seen that the system exhibits hysteresis for this set of parameters. If $\sigma$ was lowered again, the solution could stay in the QH-mode until the transcritical bifurcation at $\sigma_{\mathrm{tc} 2}=1$, where it would sharply jump to the L-mode.

\section{The smooth transition}

There are two conditions on the parameters for obtaining a smooth transition:

(a) Ensuring that the T-equilibrium never enters the phase space $\mathcal{M}$ requires $\mu_{2}>\mu_{2,0}$. (a)

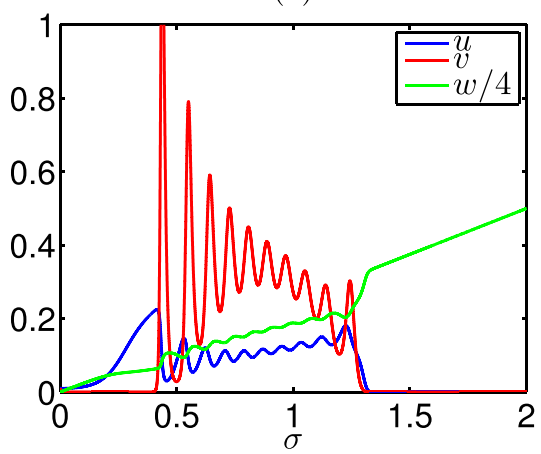

(b)

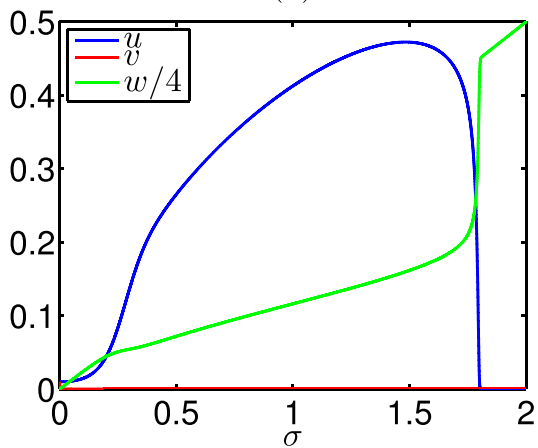

(c)

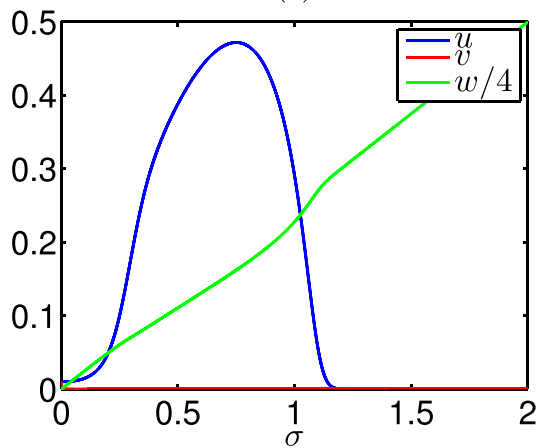

FIG. 5. Numerical solutions of the system (2) with the initial condition $u(0)=v(0)=w(0)=0.01$ and $\sigma(s)=0.01 s$ showing the three distinct types of transitions: (a) an oscillating transition where $\mu_{2}=0.08, \mu_{3}=2.8$; (b) a sharp transition where $\mu_{2}=0.4, \mu_{3}=2.8$; (c) a smooth transition where $\mu_{2}=0.4, \mu_{3}=1 / 3$. For all three plots $\mu_{1}=18, \mu_{4}=1.8$, and $\mu_{5}=8$. 
(b) Ensuring that the saddle-node bifurcation occurs outside the phase space $\mathcal{M}$ requires $\mu_{3} \leq 1 / 3$.

Fig. 4(c) shows a bifurcation diagram with these conditions fulfilled. Here, $\mu_{3}=1 / 3$ such that the saddle-node bifurcation coincides with the QH-equilibrium creating a pitchfork bifurcation at $\sigma_{\mathrm{pf}}=1$ marked with PF. The $\mathrm{H}$-equilibrium has negative $u$-coordinate, so only the $\mathrm{L}$ - and $\mathrm{QH}$-equilibrium is seen in the diagram. Before the bifurcation the $\mathrm{QH}$-equilibrium is unstable and the $\mathrm{L}$ - and $\mathrm{H}$-equilibrium are stable, while after the bifurcation the QH-equilibrium is stable, so the pitchfork bifurcation is supercritical. Fig. 5(c) shows a corresponding numerical solution to the system (2). It is seen that the transition from the L-mode to the QH-mode is completely smooth and occurs without zonal flow generation.

\section{DIMENSION REDUCTION OF THE L-H TRANSITION MODEL}

For systems with slow and fast time scales it is possible to separate the fast and slow dynamics and construct a reduced model for the slow dynamics after the fast transients have died out. We will show that the L-H transition model (2) is of slow-fast type and that the system can be reduced to be a 2-ODE system in the slow variables which captures the essential dynamics of the system.

\section{A. Geometric singular perturbation theory of slow-fast systems}

Here we briefly review geometric singular perturbation theory ${ }^{15,16}$ which forms the basis of the dimension reduction. Consider the $m+n$-dimensional system

$$
\begin{aligned}
\varepsilon \dot{x} & =\varepsilon \frac{d x}{d \tau}=f(x, y ; \varepsilon), \quad x \in \mathbb{R}^{m}, \\
\dot{y} & =\frac{d y}{d \tau}=g(x, y ; \varepsilon), \quad y \in \mathbb{R}^{n},
\end{aligned}
$$

where $0<\varepsilon \ll 1$ is a small parameter which represent the ratio between slow and the fast time scales. The variables $x$ are fast and the variables $y$ are slow. In the limit $\varepsilon=0$ we have the reduced system

$$
\begin{array}{ll}
0=f(x, y ; 0), & x \in \mathbb{R}^{m}, \\
\dot{y}=g(x, y, 0), & y \in \mathbb{R}^{n},
\end{array}
$$

where the first equation defines the $n$-dimensional critical manifold

$$
\mathcal{M}_{0}=\left\{(x, y) \in \mathbb{R}^{n} \times \mathbb{R}^{m} \mid f(x, y ; 0)=0\right\} .
$$

For the system (22) the dynamics is constrained to evolve on this manifold, governed by the differential equation (22b). Geometric singular perturbation theory provides the mathematical foundation for allowing such a reduction also for $\varepsilon>0$. Specifically, close to points $(x, y)$ on the critical manifold where the Jacobian matrix $\mathcal{D}_{x} f(x, y, 0)$ has eigenvalues with negative real parts, there is an attracting invariant manifold $\mathcal{M}_{\varepsilon}$, the slow manifold, for the system (21), as long as $\varepsilon$ is sufficiently small. Furthermore, an asymptotic expansion of the slow manifold

$$
\mathcal{M}_{\varepsilon}=\mathcal{M}_{0}+\varepsilon \mathcal{M}_{1}+\varepsilon^{2} \mathcal{M}_{2}+\cdots
$$

can be obtained from Eq. (21).

\section{B. The critical manifold}

If we define $\varepsilon=1 / \mu_{5}$ the system (2) can be recast in the form (21)

$$
\begin{aligned}
&\left(\begin{array}{c}
\dot{u} \\
\dot{v}
\end{array}\right)=\left(\begin{array}{l}
f(u, v, w) \\
g(u, v, w)
\end{array}\right)=\left(\begin{array}{c}
u\left(w-u-v-w^{4}\right) \\
\mu_{1} v\left(\frac{u}{1+\mu_{4} w^{4}}-\mu_{2}\right)
\end{array}\right) \\
& \varepsilon \dot{w}=h(u, v, w)=\sigma-w\left(1+\mu_{3} u\right) .
\end{aligned}
$$

The reduced system is

$$
\begin{aligned}
\left(\begin{array}{l}
\dot{u} \\
\dot{v}
\end{array}\right) & =\left(\begin{array}{l}
f(u, v, w) \\
g(u, v, w)
\end{array}\right) \\
0 & =h(u, v, w) .
\end{aligned}
$$

The critical manifold $\mathcal{M}_{0}$ is defined by $h(u, v, w)=0$ which can be explicitly solved for $w$

$$
w=\varphi_{0}(u, v)=\frac{\sigma}{1+\mu_{3} u} .
$$

The Jacobian matrix is simply

$$
\frac{d h}{d w}=-\left(1+\mu_{3} u\right)
$$

Since $d h / d w<0$ everywhere, geometrical singular perturbation theory yields an invariant attracting manifold $\mathcal{M}_{\varepsilon}$ close to the critical manifold $\mathcal{M}_{0}$ as long as $\varepsilon$ is sufficiently small. To lowest order in $\varepsilon$ the dynamics on the slow manifold is captured by the reduced system

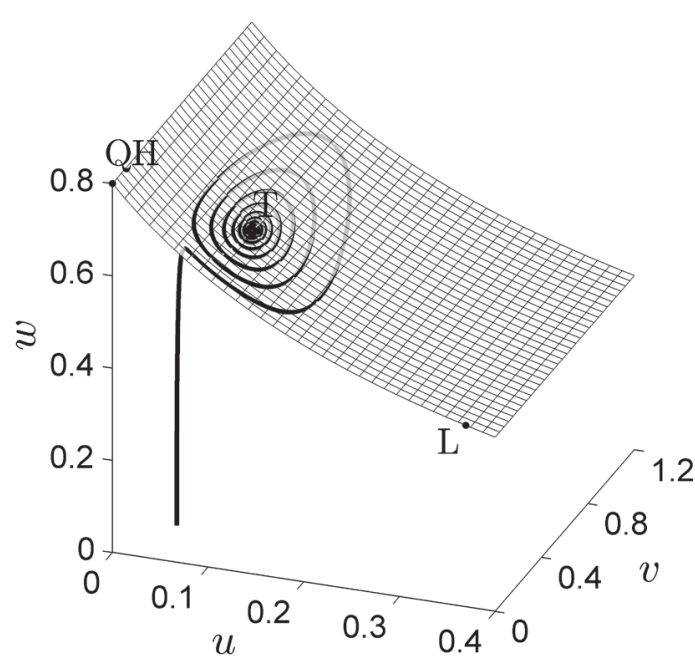

FIG. 6. The critical manifold $\mathcal{M}_{0}$ and a numerical solution of the system (2) with the initial condition $u(0)=v(0)=w(0)=0.06$ and the parameter values $\mu_{1}=18, \mu_{2}=0.08, \mu_{3}=2.8, \mu_{4}=1.8, \varepsilon=0.125$, and $\sigma=0.8$. 


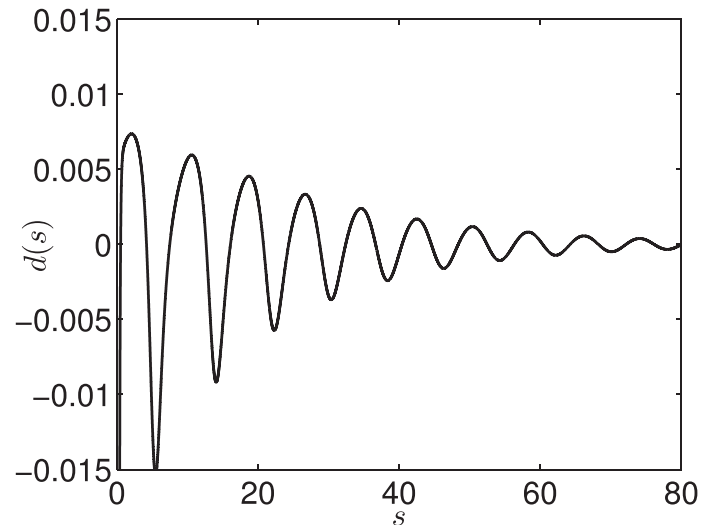

FIG. 7. The relative deviation between the critical manifold $\mathcal{M}_{0}$ and the solution shown in Fig. 6.

$$
\begin{gathered}
\dot{u}=f\left(u, v, \varphi_{0}(u, v)\right), \\
\dot{v}=g\left(u, v, \varphi_{0}(u, v)\right), \\
w=\varphi_{0}(u, v) .
\end{gathered}
$$

Fig. 6 shows a plot of the critical manifold $\mathcal{M}_{0}$ and the trajectory of a numerical solution. As a measure of the relative deviation between a solution and the critical manifold $\mathcal{M}_{0}$ define $d(s)$ to be the relative deviation between the $w$-coordinate of a solution $(u(s), v(s), w(s))$ and the corresponding $w$ coordinate on the critical manifold $\varphi_{0}(u(s), v(s))$

$$
d(s)=\frac{w(s)-\varphi_{0}(u(s), v(s))}{w(s)} .
$$

Fig. 7 shows the evolution of the relative deviation $d(s)$ between the solution and the critical manifold $\mathcal{M}_{0}$ in Fig. 6. We see that a very small deviation is obtained.

For a time-dependent $\sigma$ numerical solutions was computed at fixed values of $\varepsilon$ in the interval $\varepsilon=1 / \mu_{5} \in[0,1]$. The initial power input $\sigma(0)$ was chosen to be positive to eliminate the large relative deviation that arises when $w$ is very small. The maximal relative deviation, $\max _{s \in[0,150]}|d(s)|$, was computed for each fixed value of $\varepsilon$. Fig. 8 shows the maximal relative deviation as a function of $\varepsilon$. For $\varepsilon=0$ the

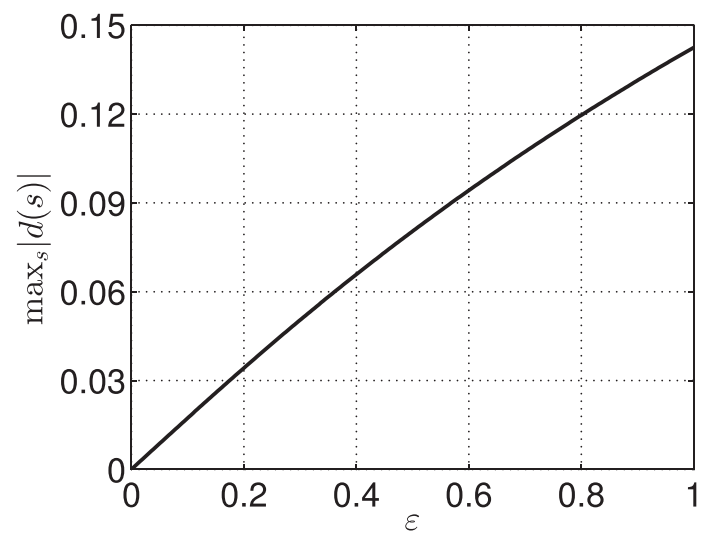

FIG. 8. Maximal relative deviation as a function of $\varepsilon$ for the parameter values $\mu_{1}=18, \mu_{2}=0.08, \mu_{3}=2.8, \mu_{4}=1.8$, and $\sigma=0.1+0.01 s, s \in[0,150]$ and the initial condition $(u(0), v(0), w(0))=\left(0.01,0.01, \varphi_{0}(0.01,0.01)\right)$. solution stays on the critical manifold for all time, and the value of $\max _{s}|d(s)|$ increases with $\varepsilon$. However, the deviation is still small for quite large $\varepsilon$, indicating that the identification of $1 / \mu_{5}$ as a small parameter is very robust.

A better approximation of the critical manifold can be obtained by including the first-order term in the expansion (23). This typically improves the deviation $d$ by a factor 10 , yielding a significantly better quantitative approximation of the original system. ${ }^{14}$ However, the qualitative dynamics changes very little, and for the present purpose the added complexity of the improved approximation does not seem worthwhile.

\section{The reduced $\mathrm{L}-\mathrm{H}$ transition model}

The analysis above establishes that the dynamics of the system (2) is essentially 2-dimensional. By restricting the system (2) to the dynamics on the critical manifold $\mathcal{M}_{0}$, we obtain the reduced L-H transition model (27), which also can be written as

$$
\begin{gathered}
\left(\begin{array}{c}
\dot{u} \\
\dot{v}
\end{array}\right)=\left(\begin{array}{c}
u\left(w-u-w^{4}-v\right) \\
\mu_{1} v\left(\frac{u}{1+\mu_{4} w^{4}}-\mu_{2}\right)
\end{array}\right), \\
w=\frac{\sigma}{1+\mu_{3} u} .
\end{gathered}
$$

The reduced system (28) has one less parameter than the full system (2) because the parameter $\mu_{5}$ has been eliminated by taking the limit $\mu_{5} \rightarrow \infty$. The phase space of the system (28) is $\mathcal{M}=\mathbb{R}_{+}^{2}$. It can be shown that numerical solutions of the reduced system look nearly identical to corresponding solutions of the full system. From a full analysis ${ }^{14}$ of the reduced system (28) it was found that all equilibrium points are the same as in the full system. The Andronov-Hopf bifurcation point has moved its position a little for the reduced system compared to the full system while all other bifurcation points are unchanged. Therefore, the bifurcation diagrams also look very similar and the same three types of transitions can be obtained.

\section{CONCLUSION}

A full bifurcation analysis of the 3-ODE L-H transition model formulated in Ref. 7 has been carried out. The model was found to contain each of the three different types of transitions; i.e., the oscillating, the sharp, and the smooth transition. The precise conditions on the parameters for obtaining each transition type were determined. The sharp transition with hysteresis and the smooth transition originated from the unfolding of a pitchfork bifurcation, while the oscillating transition resulted from an Andronov-Hopf bifurcation. Both the sharp and the smooth transition occurred without generation of zonal flow.

By using geometric singular perturbation theory the system was recognized as having slow-fast dynamics, and the system quickly converged to the dynamics of the slow subsystem. The reduced 2-ODE system resulting from the restriction of the 3-ODE system to the dynamics on the critical manifold was determined to qualitatively contain all the 
same dynamics as the full system. This means that all the dynamics in the system is essentially 2-dimensional, showing that a 2-ODE system is sufficient for obtaining a minimal model of the L-H transition. This suggests that the pressure gradient $\mathcal{N}$ is practically slaved to a combination of the drift wave turbulence level $\mathcal{E}$ and the input power $Q$.

${ }^{1}$ P. H. Diamond, S.-I. Itoh, K. Itoh, and T. S. Hahm, Plasma Phys. Controlled Fusion 47, R35 (2005).

${ }^{2}$ P. H. Diamond, A. Hasegawa, and K. Mima, Plasma Phys. Controlled Fusion 53, 124001 (2011).

${ }^{3}$ F. Wagner, Plasma Phys. Controlled Fusion 49, B1 (2007).

${ }^{4}$ F. Wagner, G. Becker, K. Behringer, D. Campbell, A. Eberhagen, W. Engelhardt, G. Fussmann, O. Gehre, J. Gernhardt, G. v. Gierke, G. Haas, M. Huang, F. Karger, M. Keilhacker, O. Klüber, M. Kornherr, K. Lackner, G. Lisitano, G. G. Lister, H. M. Mayer, D. Meisel, E. R. Müller, H. Murmann, H. Niedermeyer, W. Poschenrieder, H. Rapp, H. Röhr, F. Schneider, G. Siller, E. Speth, A. Stäbler, K. H. Steuer, G. Venus, O. Vollmer, and Z. Yü, Phys. Rev. Lett. 49, 1408 (1982).

${ }^{5}$ L. Schmitz, L. Zeng, T. L. Rhodes, J. C. Hillesheim, E. J. Doyle, R. J. Groebner, W. A. Peebles, K. H. Burrell, and G. Wang, Phys. Rev. Lett. 108, 155002 (2012).
${ }^{6}$ G. S. Xu, L. M. Shao, S. C. Liu, H. Q. Wang, B. N. Wan, H. Y. Guo, P. H. Diamond, G. R. Tynan, M. Xu, S. J. Zweben, V. Naulin, A. H. Nielsen, J. J. Rasmussen, N. Fedorczak, P. Manz, N. Yan, R. Chen, B. Cao, L. Chen, L. Wang, W. Zhang, and X. Z. Gong, "Study of L-I-H transition with a new dual gas puff imaging system in EAST superconducting tokamak," Nucl. Fusion (in press).

${ }^{7}$ E.-J. Kim and P. H. Diamond, Phys. Plasmas 10, 1698 (2003); Phys. Rev. Lett. 90, 185006 (2003).

${ }^{8}$ M. A. Malkov and P. H. Diamond, Phys. Plasmas 16, 012504 (2009).

${ }^{9}$ H. Zhu, S. C. Chapman, and R. O. Dendy, Phys. Plasmas 20, 042302 (2013).

${ }^{10}$ K. Miki and P. H. Diamond, Nucl. Fusion 51, 103003 (2011).

${ }^{11}$ K. Miki, P. H. Diamond, O. D. Guercan, G. R. Tynan, T. Estrada, L. Schmitz, and G. S. Xu, Phys. Plasmas 19, 092306 (2012).

${ }^{12}$ H. Zohm, Phys. Rev. Lett. 72, 222 (1994).

${ }^{13}$ W. Weymiens, H. J. de Blank, G. M. D. Hogeweij, and J. C. de Valenca, Phys. Plasmas 19, 072309 (2012).

${ }^{14} \mathrm{M}$. Dam, "Bifurcation analysis of low-dimensional models for L-H transition in magnetically confined plasma," M.S. thesis (Technical University of Denmark, 2013).

${ }^{15}$ N. Fenichel, J. Differ. Equations 31, 53 (1979).

${ }^{16}$ M. Desroches, J. Guckenheimer, B. Krauskopf, C. Kuehn, H. M. Osinga, and M. Wechselberger, SIAM Rev. 54, 211 (2012). 